\title{
Prediction of Insulation Capability for Ground Fault to Consider Asymmetry in $\mathrm{SF}_{6}$ Circuit Breaker
}

\author{
Yeon-Ho $\mathrm{Oh}^{\dagger}$, Ki-Dong Song*, Hong-Kyu Kim*, Hae June Lee** and \\ Sung-Chin Hahn***
}

\begin{abstract}
Currently, most high-voltage gas circuit breakers (CBs) include asymmetrical geometries in the shield, the tank, the hot-gas exhaust, and the connection parts for bushings. For this reason, a 3-dimensional (3-D) analysis of the insulation capability is necessary, rather than a 2-D analysis. However, a 3-D analysis has difficulties due to the computational time and complex modeling. This paper presents a 3-D analysis considering the asymmetry in high-voltage gas CBs and a technique to reduce the calculation time. In the proposed technique, the arc plasma requiring the most computational time is first calculated by a 2-D analysis. Then, the results such as pressure, temperature, and velocity are input as a source for the 3-D analysis. This technique is applied to a $145 \mathrm{kV}$ self-blast-type $\mathrm{CB}$ and the analysis result exhibits good agreement with the experimental result.
\end{abstract}

Keywords: Circuit breaker, 3-D analysis, Ground fault, Insulation capability, SF6, Arc plasma

\section{Introduction}

When an arc plasma is extinguished just after a fault current interruption at current zero $(\mathrm{CZ})$ of alternating current, hot gas, which is several thousand degrees, fills the inside of the gas CB. Because of the rapid reduction in the insulation capability due to the hot gas, the probability of a ground fault is increased during the transient recovery voltage (TRV) after the interruption.

Thus, the insulation capability for a ground fault must be considered in the dielectric design of a high-voltage CB [1], [2]. A 3-D analysis should be performed to estimate the dielectric characteristic after current interruption if the $\mathrm{CB}$ has an asymmetric region such as a shield, a tank, a hot-gas exhaust, or the connection parts of a bushing, as shown in Fig. 1.

In general, the calculation time of the 3-D analysis of an arc plasma depends on the accuracy of the physical arc models. The physical phenomena in a gas $\mathrm{CB}$, which are the radiative heat transfer, turbulence effects, electromagnetic field, metal vapor, nozzle ablation, and so on, are mainly focused on the arc physics [1]. However, the 3-D analysis has difficulty simulating these arc phenomena because of the significant computational time compared to a 2-D analysis.

$\dagger$ Corresponding Author: Korea Electrotechnology Research Institute, Korea. (yhoh@keri.re.kr)

* Korea Electrotechnology Research Institute, Korea. ( $\{$ kdsong, kimhk\}@keri.re.kr)

** Dept. of Electrical Engineering, Pusan University, Korea. (haejune@pusan.ac.kr)

*** Dept. of Electrical Engineering, Dong-A University, Korea. (schahn@dau.ac.kr)

Received: June 10, 2014; Accepted: May 13, 2015
This paper presents a novel technique to reduce the computational time of a 3-D analysis.

In this study, the calculation of the arc plasma requires the most computational time and is first conducted by a 2-D axisymmetrical analysis. These 2-D analysis results such as pressure, temperature, and velocity are used as an input source for the 3-D analysis. Finally, the 3-D analysis of the insulation capability is calculated for the entire domain, except the arc plasma region. For the effects of turbulent flow, the standard $\kappa-\varepsilon$ model is considered. The commercial computational fluid dynamic (CFD) code ANSYS-Fluent is used for simulation of these analyses.

In order to verify the proposed technique, experimental data for a $145 \mathrm{kV}$ self-blast-type CB is used. The analysis result exhibits good agreement with the experimental result.

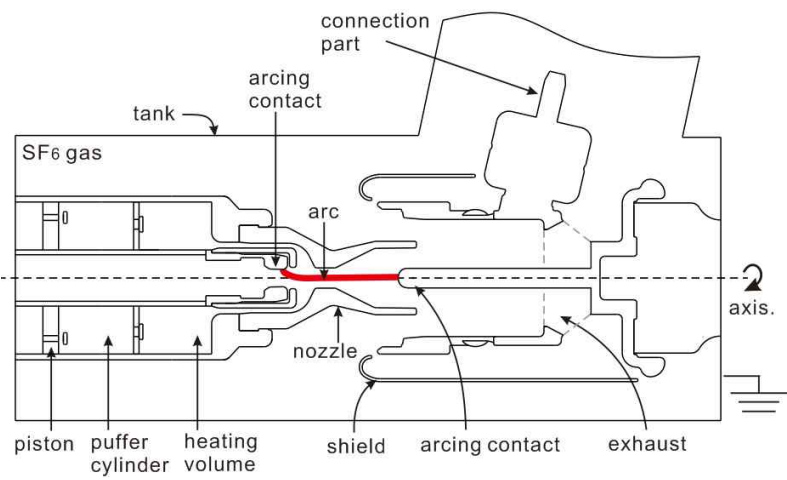

Fig. 1. Schematic diagram for a gas CB. The shield, exhaust, and connection parts are asymmetrical parts in general. 


\section{Transient Behavior of the Thermal Fluid Dynamics}

\subsection{Governing equations and arc model}

In a gas $\mathrm{CB}$, a switching arc is generated when the arcing contacts are separated, heating the surrounding insulation gas such as $\mathrm{SF}_{6}$. To extinguish the arc, hot gas between the arcing contacts should be cooled down to recover the insulation capability. For this purpose, the gas inside the puffer cylinder is compressed mechanically and blasted into the arc to exhaust the hot gas. In order to simulate the transient thermal behavior, the commercial CFD code ANSYS-Fluent is used with a combination of arc physics.

The thermal plasma can be treated as a one-temperature fluid model because of the assumption of local thermodynamic equilibrium [3].

The governing equations for analyzing the transient thermal fluid dynamics in the $\mathrm{CB}$ are represented by the mass, momentum, and energy conservation:

$$
\begin{gathered}
\frac{\partial \rho}{\partial t}+\nabla \cdot(\rho \vec{v})=S_{m} \\
\frac{\partial}{\partial t}(\rho \vec{v})+\nabla \cdot(\rho \vec{v} \vec{v})=-\nabla p+\nabla \cdot(\overline{\bar{\tau}}) \\
\overline{\bar{\tau}}=\mu\left[\left(\nabla \vec{v}+\nabla \vec{v}^{T}\right)-\frac{2}{3} \nabla \cdot \vec{v} I\right] \\
\rho \frac{\partial h}{\partial t}+\vec{v} \cdot \nabla(\rho h)=\nabla \cdot(\rho \vec{v})+\nabla \cdot(k \cdot \nabla T)+S_{e} \\
S_{e}=\sigma E^{2} \cdot R(T, r)
\end{gathered}
$$

where $\rho$ is the density, $\vec{v}$ is the velocity, $S_{m}$ is the mass source, $p$ is the pressure, $\overline{\bar{\tau}}$ is the stress tensor, $\mu$ is the molecular viscosity, $I$ is the unit tensor, $h$ is the enthalpy, $k$ is the thermal conductivity, $S_{e}$ is the energy source, $\sigma$ is the electrical conductivity, $E$ is the electric field strength, $R$ is the radiation loss, and $T$ and $r$ are the gas temperature and arc radius, respectively.

To consider the compressible effect and the turbulent model included in the real configuration of the nozzle and exhaust, the standard $\kappa-\varepsilon$ turbulence model is adopted in this work [4].

The arc energy is considered to be ohmic heating equal to $\sigma E^{2}$. With a finite element method (FEM), which was developed by using the user-defined-function of ANSYSFluent, $E$ was obtained in this study. In this work, the radiative heat transfer is treated as a loss of arc energy. The radiation loss can be calculated by using empirical arc model as function of both arc temperature and arc radius. In this model, the emitted energy from arc is $90 \%$ of the total arc energy and $80 \%$ of the emitted energy is reabsorbed in the arc edge [1]. This simplified model can save the computational time significantly.

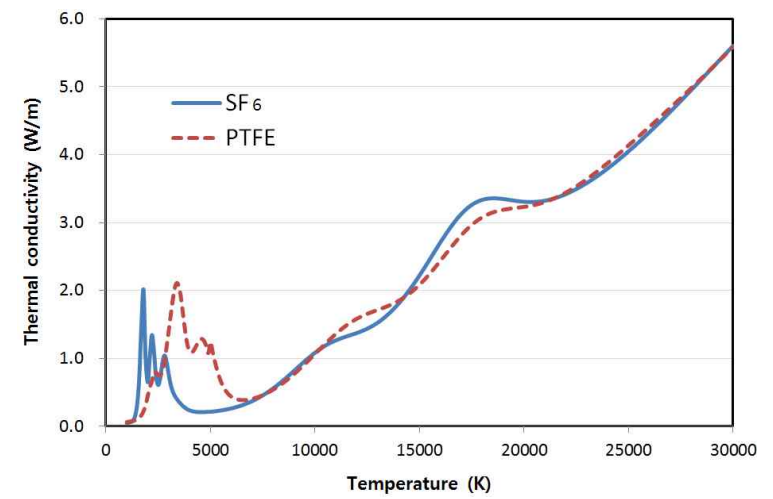

Fig. 2. Thermal conductivity of $\mathrm{SF}_{6}$ gas according to temperature.

The deviation of the insulation gas from ideal behavior was also considered by using the thermodynamic and transport properties of the gas. Fig. 2 shows the thermodynamic conductivity of a insulation medium of $\mathrm{SF}_{6}$; further details are presented in [5].

\subsection{Self-Blast-type circuit breaker and nozzle ablation}

Fig. 1 shows a schematic diagram of the geometry of the $145 \mathrm{kV}$ self-blast-type $\mathrm{CB}$, which consists of two almost symmetrical parts separated by the throat of the back-flow channel. In a self-blast-type $\mathrm{CB}$, the nozzle ablation plays an important role in the pressure increase in the heating volume, which strongly affects the interruption capability. During the high-current phase, the intense radiation of the high-temperature arc leads to ablation of the nozzle surfaces. This ablated material flows back into the heating volume during the arc, clogging the nozzle throat. The backflow of the ablation gas creates a very high pressure in the heating volume and blows hot gas between the arcing contacts.

In this study, a simplified model based on the two-zone model is suggested by the following procedure [6].

1) The total flux of ablated mass $\dot{m}_{T}$, in accordance with the arc energy, is calculated by

$$
\dot{m}_{T}=\delta \cdot U \cdot i
$$

where $\delta$ is the total ablation factor and has a value of approximately $17 \mathrm{mg} / \mathrm{kJ}, U$ is the arc voltage, and $i$ is the arc current.

2) The ablated materials, which are composed mainly of PTFE $\left(\mathrm{C}_{2} \mathrm{~F}_{4}\right)$, are mixed with cold $\mathrm{SF}_{6}$ gas. The PTFE mass fraction can be calculated by an additional massfraction equation:

$$
\frac{\partial}{\partial t}\left(\rho \alpha_{P T F E}\right)+\nabla \cdot\left(\rho v \alpha_{P T F E}\right)=\nabla \cdot\left(\Gamma \nabla \alpha_{P T F E}\right)+S_{m}(7)
$$

where $\alpha_{\text {PTFE }}$ is the PTFE mass fraction, and $\Gamma$ is the 
diffusion coefficient of PTFE, which is approximately $10^{-4} \mathrm{~kg} \mathrm{~m}^{-1} \mathrm{~s}^{-1}$.

The thermodynamic and transport properties of a mixture of these gases are given in [5].

\section{Calculation of the Dielectric Characteristics}

\subsection{Critical electric field, $E_{c r i t}$}

The insulation capability of a gas CB under hot-gas conditions can be quantified by the ratio of the critical electric-field intensity $E_{c r i t}$ to the electric-field intensity for the TRV $E_{t r v}$. $E_{c r i t}$ is the dielectric strength of the $\mathrm{SF}_{6}$ gas in accordance with the temperature and pressure. Breakdown can occur when $E_{t r v}$ is greater than $E_{c r i t}$.

Fig. 3 shows $E_{\text {crit }}$ as a function of both temperature and pressure. $E_{\text {crit }}$ has a non-linear characteristic above approximately $800 \mathrm{~K}$ because the thermal dissociation and ionization of $\mathrm{SF}_{6}$ begin to be generated at this temperature. These thermal properties, which are characterized by drastic dissociation and ionization starting at $800 \mathrm{~K}$, especially at 1,500-2,500 K, affect the thermal conductivity, as shown in Fig. 2.

It is noted that $E_{\text {crit }}$ strongly depends on temperature but weakly depends on pressure above $2,000 \mathrm{~K}$. This indicates that the probability of dielectric breakdown is very high above $2,000 \mathrm{~K}$ at the peak of the TRV with almost no dependence on pressure. Uchii [7] assumed that $E_{\text {crit }}$ is practically zero at $3,000 \mathrm{~K}$.

For $E_{\text {crit }}$ used in this work, PTFE ablated from the nozzle is not considered. If the PTFE properties are considered, $E_{\text {crit }}$ might change. However, $E_{\text {crit }}$ of pure $\mathrm{SF}_{6}$ gas is practically used to evaluate the insulation capability for a ground fault because there is small amount of PTFE vapor between the exhaust and the tank. For the same reason, the amount of variation in $E_{c r i t}$ due to erosion of the arcing contacts is small and negligible.

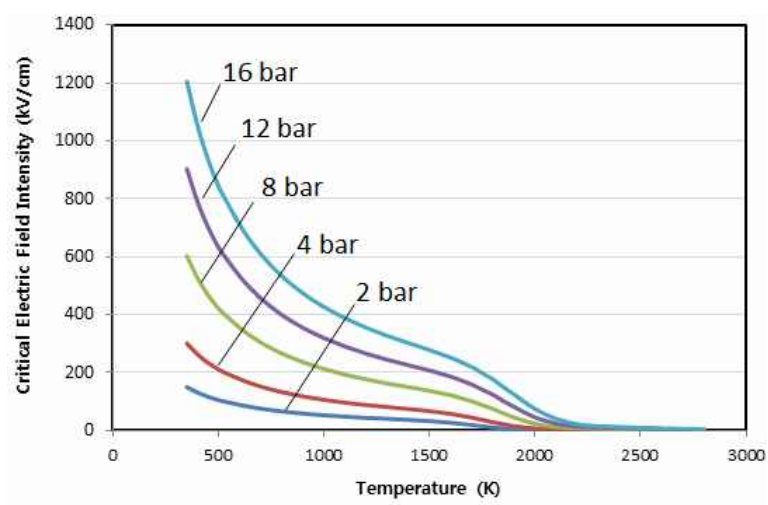

Fig. 3. Critical electric-field intensity for $\mathrm{SF}_{6}$ gas as function of both temperature and pressure.

\subsection{Algorithm for the evaluation of the dielectric characteristics}

The entire computational domain can be divided into 2D axisymmetrical and 3-D asymmetrical analyses, as shown in Fig. 4. The arc plasma region, puffer cylinder, and heating volume are included in the 2-D analysis, which are completely axisymmetrical geometries, and the 3-D analysis contains the other asymmetrical regions. The 2-D analyzed results of pressure, temperature, velocity, and density are used as the 3-D analysis input data.

Fig. 5 shows the flowchart for the prediction algorithms of the dielectric strength under hot-gas conditions.

The physical phenomena of the arc plasma can be calculated via a 2-D axisymmetrical analysis including nozzle ablation. The hot gas generated from the arc plasma flows toward the nozzle exhaust, including pressure, velocity, density and temperature components.

These 2-D output variables then are converted to 3-D input variables, and the 3-D thermal behavior and insulation capability are calculated, except the arc plasma.

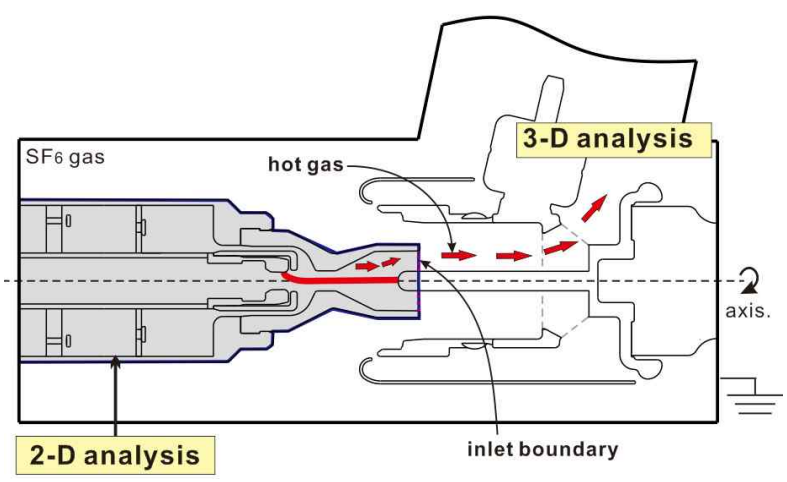

Fig. 4. Entire computational domain for the 2-D axisymmetrical and 3-D asymmetrical analysis geometries.

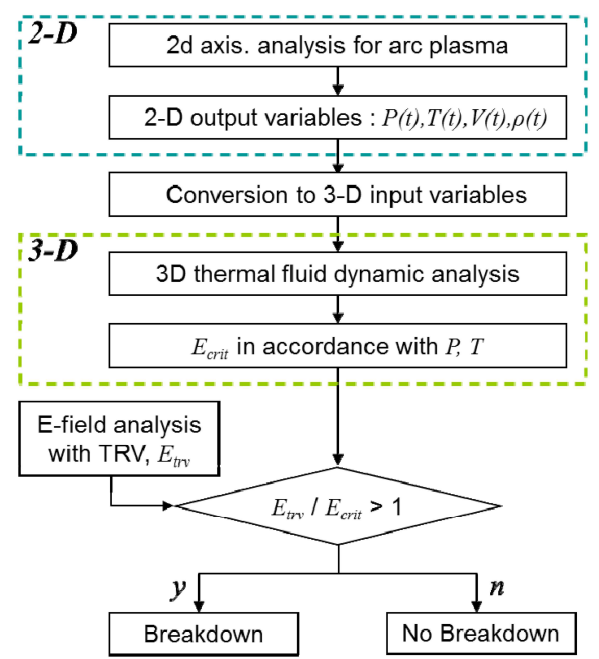

Fig. 5. Flowchart for the prediction algorithms of the dielectric strength. 


\subsection{Inlet boundary and conditions for the 3-D Analysis}

Fig. 6 shows the 2-D output and 3-D inlet boundaries in the real $\mathrm{CB}$ geometry. The pressure $P(t)_{2 d}$ from the 2-D analysis is used as the inlet source of the 3-D analysis with the temperature $T(t)_{2 d}$, velocity $v(t)_{2 d}$, and density $\rho(t)_{2 d}$ :

$$
\begin{aligned}
P(t) & =P(t)_{s_{2 d}}+P(t)_{d_{2 d}} \\
& =P(t)_{s_{2 d}}+\frac{1}{2} \rho(t)_{2 d}\left|\vec{v}(t)_{2 d}\right|^{2} \\
T(t) & =T(t)_{2 d}
\end{aligned}
$$

where $P(t)_{s_{2 d}}$ is the static pressure, and $P(t)_{d_{2 d}}$ is the dynamic pressure from the 2-D analysis. In this study, the entire computational domain except for the nozzle area is assumed to be a subsonic flow with a flow-speed Mach number less than one.

For conversion from the 2-D analysis results to the source of the 3-D analysis, there is an absence in the input dimensions due to the expansion of dimensions, namely, the z-axis components do not exist. For the 2-D analysis, the arc plasma between the arcing contacts can be fully preformed in an axisymmetrical analysis. In the nozzle exhaust, the hot gas from the arc collides with the arcing contacts and shields. Then, there could be z-axis components outside of the nozzle.

The axial and radial components in the 2-D axisymmetrical analysis are used as the sources of $x$ - and $y$-axis components, respectively, in the 3-D analysis. However, zeroes are used as the inputs for the $\mathrm{z}$ components in the 3$\mathrm{D}$ analysis owing to the absence of $\mathrm{z}$-axis components from the 2-D analysis.

Thus, the inlet boundary in 3-D should be the surface on which the z-components of the output variables, pressure and velocity, are minimized. In this study, the inlet

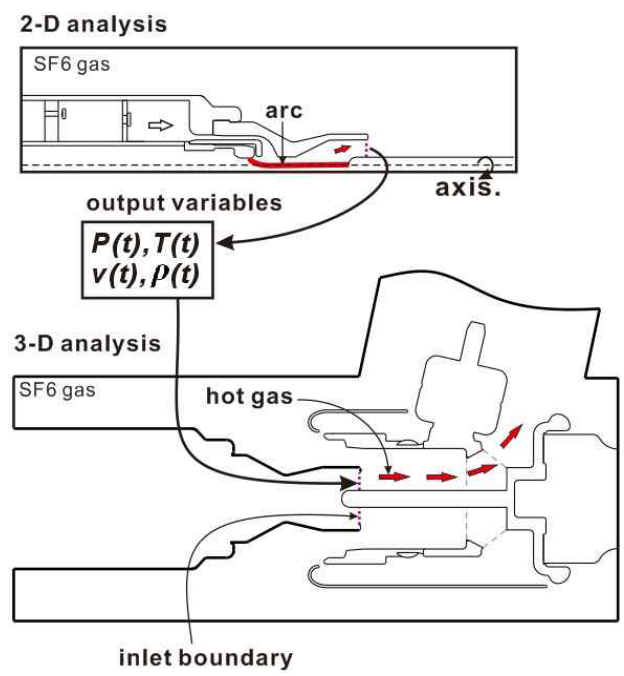

Fig. 6. Output variables and inlet boundary for the 3-D analysis. boundary in 3-D is the surface near the nozzle exhaust in Fig. 6. These $z$-components in the inlet surface are expected to be negligible because of the significantly strong axial velocity component. This assumption could be validated by a comparison with experimental results.

\section{Comparison with Experimental Results}

\subsection{5-kV Self-blast-type gas circuit breaker}

Fig. 7 shows the $145 \mathrm{kV}$ self-blast CB installed in the testing room. There are the asymmetric parts inside for the $145 \mathrm{kV}$ self-blast $\mathrm{CB}$ as shown in Fig. 8. The amplitude of the testing current is $40 \mathrm{kA}_{\mathrm{rms}}$ with a frequency of $60 \mathrm{~Hz}$. The arcing time, which is the time after the separation of the arcing contacts to $\mathrm{CZ}$, is $20.3 \mathrm{~ms}$. As a result of the test a dielectric breakdown occur between a shield and tank at 73.6 $\mu$ s after $\mathrm{CZ}$.

In general, the pressure increase inside a self-blast gas $\mathrm{CB}$ is greater than a puffer-type $\mathrm{CB}$ because the hot gas from the arc plasma flows backward, heating the volume by nozzle ablation. This heats the gas and increases the pressure inside the heating volume. Thus, there would be many weak points in the dielectric capability of a self-blast gas $\mathrm{CB}$ owing to the large amount of hot gas.

In a self-blast $\mathrm{CB}$, nozzle ablation considerably affects the pressure distribution during the high-current phase and

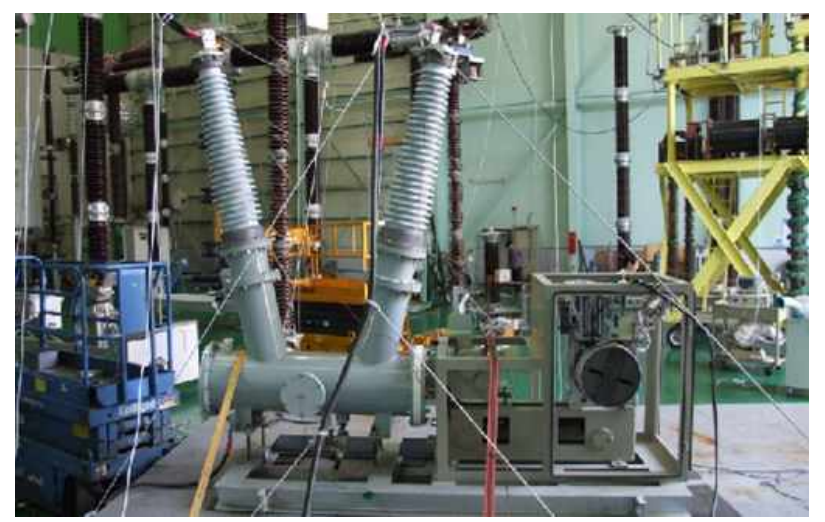

Fig. 7. Installation of a $145 \mathrm{kV}$ self-blast gas $\mathrm{CB}$ in the testing room.

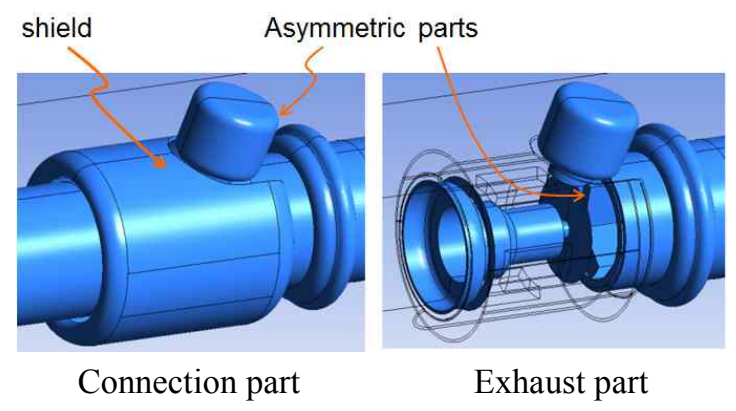

Fig. 8. Asymmetric parts for a $145 \mathrm{kV}$ self-blast CB. 
also the movement of hot gas. Considering nozzle ablation and the arc energy the movement of the contacts are simulated.
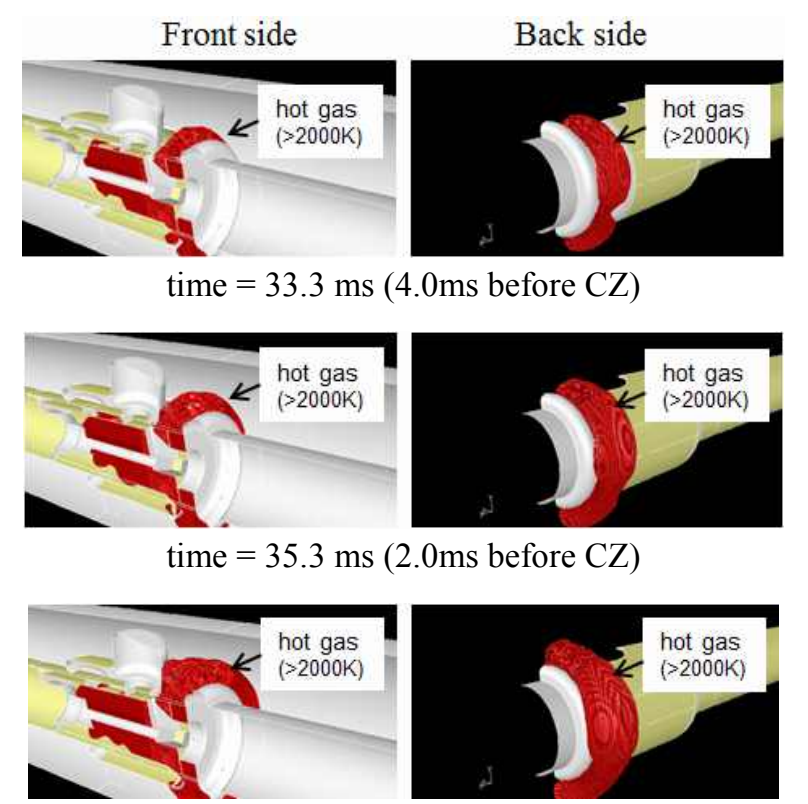

time $=37.3 \mathrm{~ms}(\mathrm{CZ})$

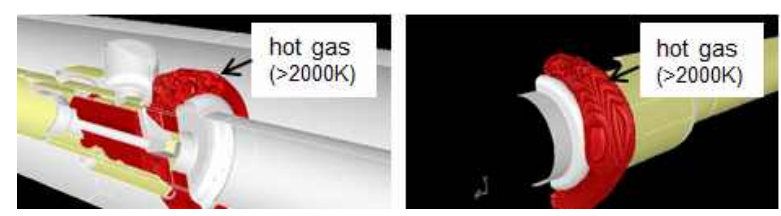

time $=37.3736 \mathrm{~ms}(73.6 \mu \mathrm{s}$ after $\mathrm{CZ})$

Fig. 9. Contours of hot gas greater than $2000 \mathrm{~K}$ before and after $\mathrm{CZ}$ for a $145 \mathrm{kV}$ self-blast gas $\mathrm{CB}$.

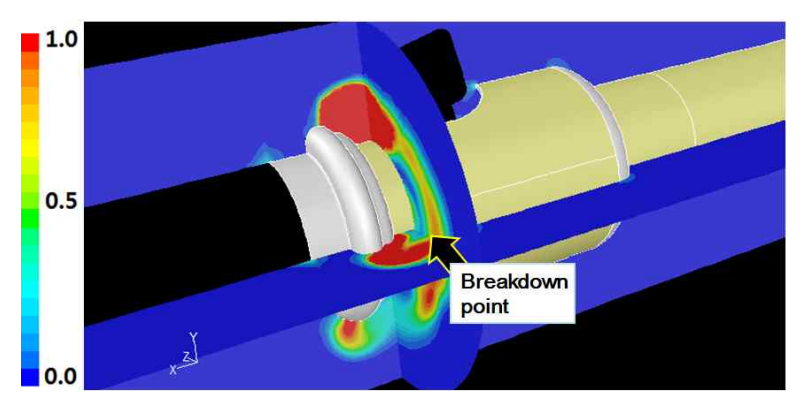

(a) Contours of $E_{t r v} / E_{\text {crit }}$

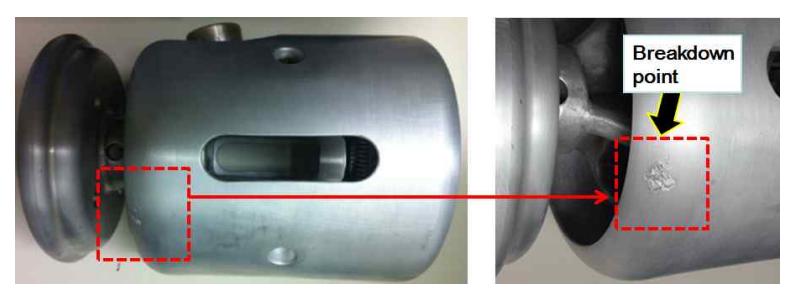

(b) The location of the breakdown

Fig. 10. Contours of $E_{t r v} / E_{c r i t}$ and the location of the breakdown.
As a result of simulation contours of the hot gas before and after $\mathrm{CZ}$ are shown in Fig. 9. After $\mathrm{CZ}$, the temperature of the hot gas is greater than $2000 \mathrm{~K}$, and there is a high probability of breakdown in the exhaust. Fig. 10 (a) and (b) show the contours of $E_{t r v} / E_{c r i t}$ and the location of the breakdown after the test respectively. The test results show that there is a dielectric breakdown at $t=73.6 \mu$ s after $\mathrm{CZ}$, and the location of the breakdown is shown in Fig. 10 (b). The location having a higher probability of breakdown, as predicted by the analysis, is similar to that of the test results. The experimental results demonstrate the reliability of the proposed technique.

The computational time including the modeling time is reduced to $48 \mathrm{~h}$ in comparison with $220 \mathrm{~h}$ for the full 3-D analysis.

It should be emphasized that $E_{\text {crit }}$ is affected by temperature rather than pressure. Thus, the design to adequately pass the hot gas or to extend the passage of hot gas from the exhaust to the outlet is necessary to decrease the temperature of the hot gas from the exhaust.

\section{Conclusion}

This paper presents a technique to characterize the insulation capability for a ground fault considering the asymmetry in a gas $\mathrm{CB}$. The results of this work are summarized as follows.

1) In order to consider the asymmetric configuration of a $\mathrm{CB}$, a 3-D analysis based on the 2-D analysis results is performed.

2) To reduce the calculation time, a 2-D axisymmetrical analysis is conducted for the physical phenomena of the arc plasma, which requires considerable computational time.

3) The output variables from the 2-D analysis are used as the source of the 3-D analysis as function of time. In the 3-D analysis, $E_{t r v} / E_{c r i t}$ is obtained as a function of pressure and temperature, and the characteristics of the dielectric strength are evaluated.

4) The simulation results are compared with experimental results and demonstrate the reliability of the proposed technique.

It is noted that the total computational time is drastically reduced in comparison with a full 3-D analysis. However, this technique does not consider the $\mathrm{z}$-axis components of the inlet boundary in the 3-D analysis. Thus, the proposed technique is only applied to a gas $\mathrm{CB}$ that has a strong axial flow.

\section{References}

[1] Hong-Kyu Kim, Jin-Kyo Chong and Ki-Dong Song, 
"Analysis of Dielecric Breakdown of Hot SF6 Gas in a Gas Circuit Breaker," JEET, vol. 5, No. 2, pp.264269,2010

[2] H. Takana, T. Uchii, H. Kawano, and H. Nishiyama, "Real-Time Numerical Analysis on Insulation Capabiity Improvement of Compact Gas Circuit Breaker," IEEE Trans. Power Delivery, vol. 22, No.3, pp.15416, July 2007.

[3] M. Yousfi, Ph. Robin-Jouan, Z. Kanzari, "Breakdown Electric Field Calculations of $\mathrm{Hot}^{\mathrm{SF}_{6}}$ for High Voltage Circuit Breaker Applications," IEEE Trans. on Dielect. Elect. Insulation, Vol. 12, No. 6, pp. 1192-1200, Mar. 2005.

[4] Ki-Dong Song, Byeong-Yoon Lee and Kyong-Yop Park, "Calculation of the Post-Arc Current in a Supersonic Nozzle by Using the $\kappa-\varepsilon$ Turbulence Model," Journal of the Korean Physical Society, vol. 45, No. 6, pp.1537-43, Dec. 2004.

[5] Jin Ling Zhang, Jiu Dun Yan, Anthony B. Murphy, W. Hall, and M.T.C. Fang, "Computational Investigation of Arc Behavior in an Auto-Expansion Circuit Breaker Contaminated by Ablated Nozzle Vapor," IEEE Trans. on Plasma Science, vol. 30, No. 2, pp. 706-19, Apr. 2002.

[6] M Claessens, K Möller and H G Thiel, "A computational fluid dynamics simulation of high- and low-current arcs in self-blast circuit breakers," $J$. Phys. D: Appl. Phys., 30, pp. 1899-1907, 1997

[7] T. Uchii, T. Nakamoto, S. Nishiwaki, M. Toyoda, and S. A. Boggs, "Optimization of Dead Tank Gas Circuit Breaker Design Based on Quantification of Hot Gas Flow and Dielecgtric Properties," IEEE Trans. Power Delivery, vol. 19, No. 1, pp. 181-85, Jan. 2004.

[8] L Müller, "Modelling of an ablation controlled arc," $J$. Phys. D:Appl. Phys., 25, pp.1253-59, 1993.

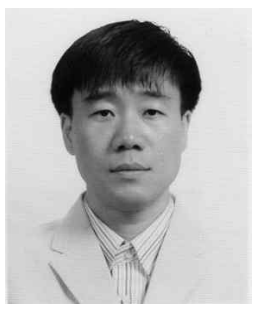

Yeon-Ho Oh He received the B.S. and M.S. degrees in electrical engineering from Dong-A University, Busan, Korea, in 1991 and 1993, respectively. He is currently a Research Engineer with the Power Apparatus Research Center, HVDC Research Division, Korea Electrotechnology Research Institute (KERI).

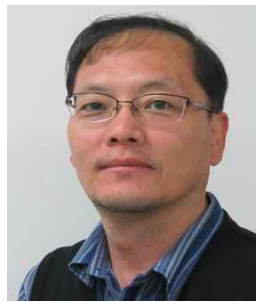

Ki-Dong Song He received the B.S. and M.S. degrees in electrical engineering from InHa University, Incheon, Korea, in 1988 and 1990, respectively. He got his Ph.D. degree in electrical engineering from Busan National University in 2003. He worked for Korea Electrotechnology Research Institute
(KERI) from 1990 and is now the director of the Power Apparatus Research Center, HVDC Research Division. His research interests focus on analysis and design for $\mathrm{AC}$ Power Devices and HVDC circuit breakers.

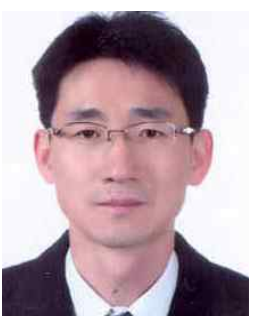

Hong-Kyu Kim $\mathrm{He}$ was born in Yeongchon, Korea, in 1969. He received his B. Sc., M. Sc. and Ph.D. degrees in 1995, 1997 and 2001, respectively, from the School of Electrical Engineering, Seoul National University, Korea. He has been working for the Korea Electrotechnology Research Institute (KERI) since 2001. His research field covers the numerical analysis of electromagnetic fields, and the analysis of cold and hot gas flow in high voltage gas circuit breakers. He is a regular member of the CIGRE working group A3.24.

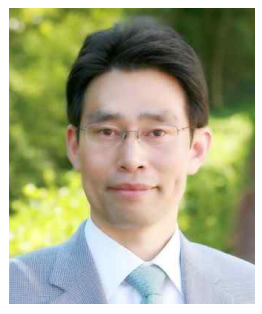

Hae June Lee Professor Hae June Lee received his B.S. degree with from the Department of Nuclear Engineering at the Seoul National University in 1994, and his M.S. and Ph.D. degrees in physics in 1996 and 1998 from POSTECH. He worked as a post doc. in the Department of Electrical Engineering at the UC Berkeley in 2000 and 2001 and as a research scientist in Korea Electrotechnology Research Institute (KERI) after then. Since 2004, Prof. Lee has been a faculty member in the Department of Electrical Engineering at Pusan National University (PNU), South Korea.

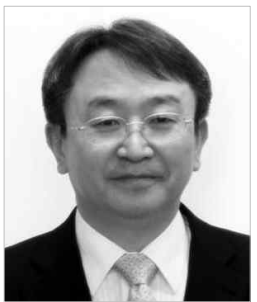

Sung-Chin Hahn He received the B.S., M.S., and Ph.D. degrees in electrical engineering from Seoul National University, Seoul, Korea, in 1979, 1981, and 1992, respectively. He is currently a Professor with the Department of Electrical Engineering, Dong-A University, Busan, Korea. His current research covers multi-physics analysis and optimal design of power apparatus and electric machines. 